\title{
Ultra Massive Passive Galaxies at $\mathrm{z} \sim 1.7$
}

\author{
Liz Arcila-Osejo ${ }^{1}$, Marcin Sawicki ${ }^{1}$, Anneya Golob ${ }^{1}$, \\ Stephane Arnouts ${ }^{2,3}$ and Thibaud Moutard ${ }^{2,3}$ \\ ${ }^{1}$ Saint Mary's University, 923 Robie Street, Halifax, N.S., Canada \\ ${ }^{2}$ Laboratoire d'Astrophysique de Marseille, Rue Frdric Joliot Curie, 13013 Marseille, France \\ ${ }^{3}$ CFHT, 65-1238 Mamalahoa Hwy, Waimea, Kamuela 96743, United States. \\ email: osejo@ap.smu.ca
}

\begin{abstract}
At redshift $\mathrm{z} \sim 1.7$ the Universe was at the peak of its star-formation activity. It is thus a puzzle why some galaxies, many of them very massive $\left(\mathrm{M}_{*} \geqslant 10^{11} \mathrm{M} \odot\right)$, had already chosen to stop forming stars. These ultra-massive galaxies, guaranteed to be the central galaxies of their host dark matter halos, must have attained very high rates of star formation to assemble their stellar masses in such a short amount of time. Using the largest (to date) K-selected gzK survey of passive galaxies (in an effective area of $\sim 27.5 \mathrm{deg}^{2}$ ) we study the demographics of these dead monsters, hoping to help understand the quenching mechanism that shut them down.
\end{abstract}

Keywords. galaxies: evolution, galaxies: high-redshift, galaxies: surveys.

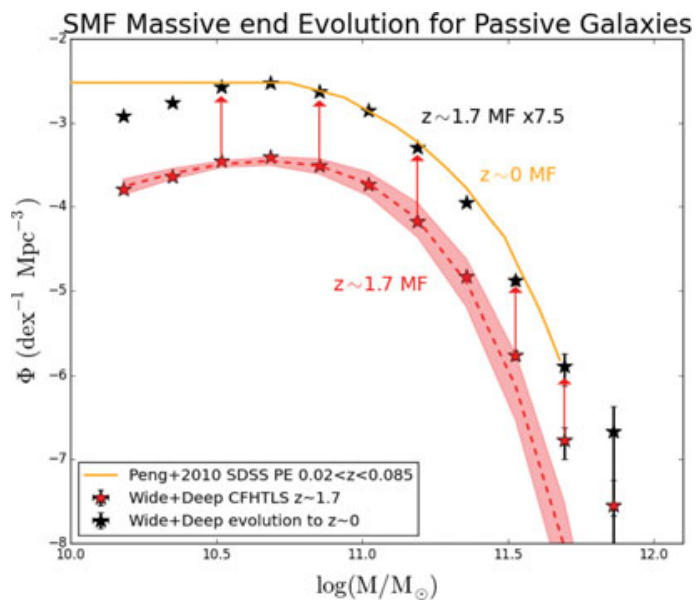

(a) A combination of the CFHTLS Deep + Wide surveys and the Vipers K-band and WIRDS surveys, allows us to construct the largest catalog of K-selected gzKs passive galaxies at $\mathrm{z} \sim 1.7$.

(b) Using this sample we are able constrain the massive end of the stellar mass function with unprecedented precision.

(c) Simple density evolution (as seen in the Figure in red arrows) of the $\mathrm{z} \sim 1.7 \mathrm{MF}$, matches the z $\sim 0$ MF (yellow curve from Peng et al. 2010, ApJ, 721, 193); the number density of galaxies increases uniformly independently of mass.

(d) This simple density evolution suggests that the mechanism that quenches starformation in massive galaxies does not change with time and indicates a lack of postquenching mechanisms that could alter $\mathrm{M}^{*}$ (e.g., mergers). 